\title{
Stabilization of Hypoxia-Inducible Factor-1 Alpha Augments the Therapeutic Capacity of Bone Marrow-Derived Mesenchymal Stem Cells in Experimental Pneumonia
}

\author{
Naveen Gupta ${ }^{1,2 *}$ and Victor Nizet ${ }^{3,4}$ \\ ${ }^{1}$ Division of Pulmonary and Critical Care, Department of Medicine, School of Medicine, University of California, San Diego, \\ La Jolla, CA, United States, ${ }^{2}$ Department of Molecular Medicine, The Scripps Research Institute, La Jolla, CA, United States, \\ ${ }^{3}$ Division of Host-Microbe Systems and Therapeutics, Department of Pediatrics, School of Medicine, University of California, \\ San Diego, La Jolla, CA, United States, ${ }^{4}$ Skaggs School of Pharmacy and Pharmaceutical Sciences, School of Medicine, \\ University of California, San Diego, La Jolla, CA, United States
}

OPEN ACCESS

Edited by:

Jesús Gonzalo-Asensio, Universidad de Zaragoza, Spain

Reviewed by: Paschalis Sidéras, Biomedical Research Foundation of the Academy of Athens, Greece Anna Krasnodembskaya, Queen's University Belfast, United Kingdom

${ }^{*}$ Correspondence: Naveen Gupta n6gupta@ucsd.edu, ngupta@scripps.edu

Specialty section:

This article was submitted to Pulmonary Medicine, a section of the journal Frontiers in Medicine

Received: 08 February 2018 Accepted: 19 April 2018

Published: 04 May 2018

Citation:

Gupta N and Nizet V (2018) Stabilization of Hypoxia-Inducible Factor-1 Alpha Augments the Therapeutic Capacity of Bone Marrow-Derived Mesenchymal Stem Cells in Experimental

Pneumonia.

Front. Med. 5:131.

doi: 10.3389/fmed.2018.00131
Bone marrow-derived mesenchymal stem cells (MSCs) have therapeutic effects in experimental models of lung injury. Hypoxia-inducible factor-1 alpha ( $\mathrm{HIF-1 \alpha})$ is a transcriptional regulator that influences cellular metabolism, energetics, and survival under hypoxic conditions. The current study investigated the effects of stabilizing HIF- $1 \alpha$ on the therapeutic capacity of MSCs in an experimental mouse model of bacterial pneumonia. HIF-1 $\alpha$ stabilization was achieved by the small molecule prolyl-hydroxlase inhibitor, AKB4924 (Aerpio Therapeutics, Inc.), which blocks the pathway for HIF-1 $\alpha$ degradation in the proteosome. In vitro, pre-treatment with AKB-4924 increased HIF-1 $\alpha$ levels in MSCs, reduced the kinetics of their cell death when exposed to cytotoxic stimuli, and increased their antibacterial capacity. In vivo, AKB-4924 enhanced MSC therapeutic capacity in experimental pneumonia as quantified by a sustainable survival benefit, greater bacterial clearance from the lung, decreased lung injury, and reduced inflammatory indices. These results suggest that HIF- $1 \alpha$ stabilization in MSCs, achieved ex vivo, may represent a promising approach to augment the therapeutic benefit of these cells in severe pneumonia complicated by acute lung injury.

Keywords: mesenchymal stem cells, hypoxia-inducible factor-1 alpha, lung injury, pneumonia, sepsis

\section{INTRODUCTION}

Severe pneumonia is the most common cause of sepsis and respiratory failure among critically ill patients. The mortality in the most severe cases can approach $50 \%$, and treatment options have become increasingly limited due to the rapid emergence of multi-drug resistant bacterial strains, particularly among enteric Gram-negative bacteria (1-3). New treatment options that can harness the potential of the innate immune system are needed to more effectively manage this complex condition.

Bone marrow-derived mesenchymal stem cells (MSCs) have been studied as a potential source for cell-based therapy for a wide range of experimental organ injury models. In particular, there has been a considerable amount of focus on using MSCs as a therapy for severe lung injury 
and sepsis as there are no proven pharmacological therapies in this field (4-9). MSCs have a number of biological properties that lend them to producing a favorable outcome in lung injury and sepsis including immunomodulation, secretion of epithelial and endothelial growth factors, and augmentation of host defense to infection $(6,10,11)$. However, the clinical benefits of MSCs in trials have been modest, which may be due to a lack of sustained benefit given MSC death and clearance under inflammatory conditions in vivo. It has previously been shown that non-viable MSCs exert no therapeutic benefit (5). Thus, methods to enhance MSC survival and augment their therapeutic capacity should improve their efficacy in clinical lung injury and sepsis.

Hypoxia-inducible factor- 1 alpha $(\mathrm{HIF}-1 \alpha)$ is an important transcriptional regulator that controls many cellular processes under hypoxic conditions, and the injured lung represents a low-oxygen tension environment that presents a metabolic stress to cells introduced into that space. Prior efforts suggested that stabilization of cellular HIF-1 $\alpha$ levels could increase the therapeutic function of MSCs in cardiac and vascular injury models (12-14). Consequently, we hypothesized that HIF-1 $\alpha$ stabilization in MSCs would enhance their therapeutic efficacy in experimental lung injury and pneumonia, potentially by improving cell survival in the face of inflammatory, cytotoxic stimuli. To that end, we pharmacologically stabilized HIF-1 $\alpha$ in MSCs using AKB-4924 (Aerpio Therapeutics, Blue Ash, OH, USA) given our previous experience with the selective potency of this compound (15-17).

\section{METHODS}

\section{Isolation, Characterization, and Culturing of MSCs}

Mouse MSCs were isolated from 8- to 10-week old male C57BL/6J mice and characterized as published before (8). MSCs were then cultured using MEM-alpha media (Gibco, catalog \#12561) with 15\% FBS (Gibco, catalog \#12662-029) and 1\% Pen/Strep/ L-Glutamine and used for in vitro and in vivo experiments from passages 5 to 10 .

\section{HIF-1 $\alpha$ Stabilization in MSCs and Western Blotting}

Mesenchymal stem cells were incubated in the presence of AKB-4924 in a 12-well plates for 4 and $24 \mathrm{~h}$ to determine the optimal time and concentration for HIF-1 $\alpha$ stabilization in MSCs. AKB-4924 was used at 10 and $100 \mu \mathrm{M}$ in MEM-alpha supplemented with 5\% FBS. MSCs were then lysed and the protein fraction isolated, quantified, and analyzed for HIF- $1 \alpha$ expression by Western blotting (see Supplementary Material for details). Based on the data, AKB-4924 was used at $100 \mu \mathrm{M}$ for $4 \mathrm{~h}$ on MSCs to stabilize HIF- $1 \alpha$ in most in vitro and in vivo studies.

\section{In Vitro Bacterial Killing Studies}

To determine if AKB-4924 enhances MSC killing of bacteria, separate assays were done with live MSCs and MSC-derived conditioned media in the presence of Escherichia coli (see Supplementary Material). Mouse cathelicidin-related antimicrobial protein (CRAMP ELISA, MyBioSource, catalog \#MBS280706) was specifically measured to determine if it accounted for the antimicrobial effects induced by AKB-4924. Gene expression for CRAMP was quantified using $\mathrm{qPCR}$ as outlined below.

\section{In Vitro Cell Death and Caspase 3/7 Activity}

To measure the effect of AKB-4924 on MSC death when exposed to cytotoxic, inflammatory stimuli, studies were done to measure caspase $3 / 7$ activity in a plate-based assay (Promega, catalog \#G7790). TNF- $\alpha$ and cycloheximide were chosen as the stimuli since this combination resulted in the most reproducible quantity of cell death for MSCs, and it has been published as an in vitro method to model cell death in an inflammatory environment $(18,19)$ (see Supplementary Material).

\section{RNA Isolation and qPCR}

In vitro studies were done to determine if AKB-4924 regulated expression of selected genes (CRAMP, Oct4, TWIST) in MSCs that could account for the observed in vitro and in vivo effects. RNA was isolated and qPCR was carried out using standard procedures (see Supplementary Material).

\section{In Vivo E. coli Pneumonia Model and Experimental Design}

All mice used for these experiments were male C57BL/6J (Jackson Labs) between the ages of 10 and 15 weeks of age. All experiments were approved by the University of California, San Diego (UCSD) Institutional Animal Care and Use Committee, and mice were housed in a UCSD facility approved by the Association for Assessment and Accreditation of Laboratory Animal Care. The general experimental design that we followed is as previously published $(5,8)$ (see Supplementary Material).

\section{Assessment of Lung Injury, Inflammation, and Bacterial Burden}

Lung injury was assessed by histological methods and scored using a previously published method (20). Markers of inflammation and permeability were measured in the bronchoalveolar lavage (BAL) fluid $(5,8)$, and bacterial burden was calculated from whole lung homogenate (see Supplementary Material).

\section{Statistical Analysis}

The majority of the data is presented as mean \pm SD for each group analyzed. An unpaired, two-sided Student's $t$-test was used for comparisons between sets of data. For sets of data with a small sample size (total $n<20$ ), a Mann-Whitney $U$ test was used. If multiple groups of data were compared simultaneously, an ANOVA was used. Survival data were analyzed using a log-rank test. A $p$-value $<0.05$ was used for statistical significance for all analyses. 


\section{RESULTS}

\section{AKB-4924 Stabilizes HIF-1 $\alpha$ in MSCs and Reduces MSC Death Under Cytotoxic Conditions}

AKB-4924 stabilization of HIF-1 $\alpha$ protein levels in MSCs occurred at a concentration of 10 or $100 \mu \mathrm{M}$ and was readily apparent after $4 \mathrm{~h}$ incubation (Figure 1A). MSCs treated with AKB-4924 exhibited significantly reduced cell death, as measured by caspase $3 / 7$ activity, when exposed to TNF- $\alpha$ and cycloheximide (Figure 1B).

\section{AKB-4924 Enhances the Antibacterial Capacity of MSCs}

In vitro, AKB-4924 was able to significantly improve MSC-based reduction of viable $E$. coli. The effect of AKB-4924 occurred under both basal and TNF- $\alpha$ stimulated conditions (Figure 1C). Conditioned media from AKB-4924 stimulated and TNF- $\alpha+$ LPS stimulated MSCs demonstrated an approximate $20 \%$ reduction in viable E. coli compared with conditioned media from unstimulated MSCs (Figure 1D). This suggests that release of an antimicrobial factor into the conditioned media may account for part of the increased bacterial killing by MSCs that is induced by AKB-4924. We hypothesized that this factor may be mouse CRAMP given previous literature demonstrating that the human cathelicidin antimicrobial protein LL-37 is a potential transcriptional target of HIF- $1 \alpha$, and that human MSCs exert antibacterial effects via LL-37 secretion (10, 21). However, under the conditions utilized in this study, we did not detect a significant increase in CRAMP protein secretion in HIF- $1 \alpha$ stabilized MSCs (Figure 1E).

\section{AKB-4924 Improves MSC-Derived Therapeutic Capacity In Vivo}

To determine if the in vitro benefits with AKB-4924 described above translated into greater MSC-derived therapeutic capacity in vivo, the experimental design using an E. coli pneumonia model outlined in Figure 2A was utilized. While both unstimulated and AKB-4924 stimulated MSCs exerted significant survival benefits at $72 \mathrm{~h}$ (Figure 2B), only MSCs incubated with AKB-4924 conferred sustained protection against mortality over the course of 7 days (Figure 2C). Bacterial clearance from the lung at $24 \mathrm{~h}$ post-infection was significantly improved with MSCs incubated with AKB-4924 as well (Figure 2D). In addition, HIF- $1 \alpha$ stabilized MSCs led to a significant reduction in inflammatory indices such as BAL myeloperoxidase (MPO) and macrophage inflammatory protein-2 (MIP-2) levels $24 \mathrm{~h}$ after infection (Figures 2F,G, respectively), though there was not a significant reduction in the total BAL cell count (Figure 2E) or BAL albumin concentration
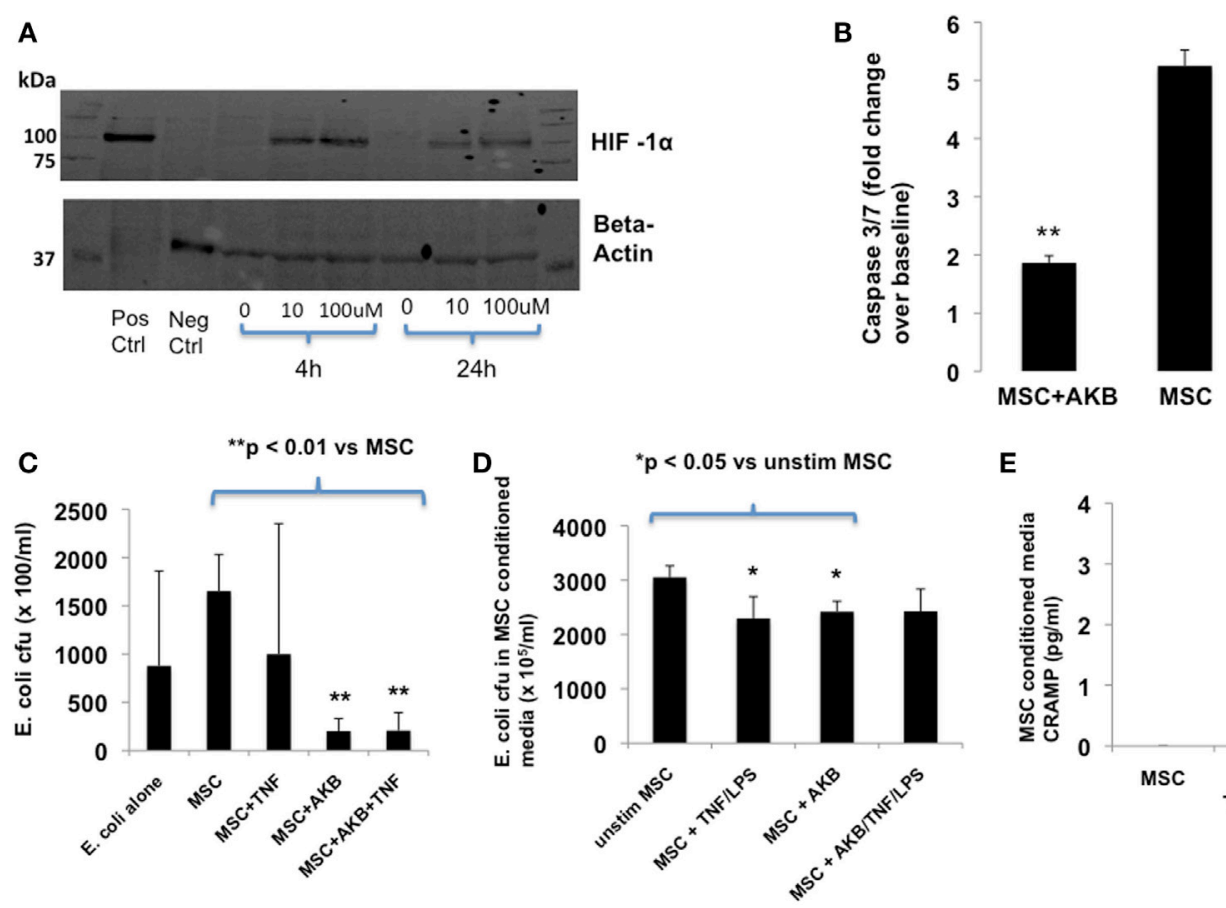

E

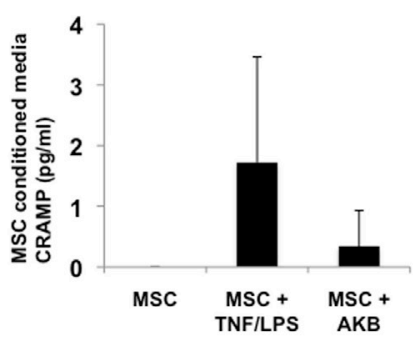

FIGURE 1 | AKB-4924 stabilizes hypoxia-inducible factor $1 \alpha$ (HIF-1 $\alpha)$ in mesenchymal stem cells (MSCs) and improves MSC survival and bacterial clearance under in vitro conditions. Use of AKB-4924 at both 10 and $100 \mu \mathrm{M}$ resulted in detectable amounts of HIF-1 $\alpha$ protein in MSCs after 4 and $24 \mathrm{~h}$ of incubation (A). AKB-4924 significantly reduced caspase 3/7 activity in MSCs exposed to TNF- $\alpha$ and cycloheximide for $5 \mathrm{~h}\left[(\mathbf{B})\right.$, ${ }^{* *} p<0.01$ for MSC + AKB vs MSC, $n=6$ per group]. MSCs pre-stimulated with AKB-4924 exhibited enhanced bacterial clearance at $6 \mathrm{~h}\left[(\mathbf{C})\right.$, ${ }^{* *} p<0.01$ when compared with MSC group, $n=4$ per group] that may be partially due to a soluble antimicrobial factor [(D), ${ }^{*} p<0.05$ compared with unstim group, $n=4$ per group]. Cathelicidin-related antimicrobial protein (CRAMP) was not significantly increased in the conditioned media of MSCs pre-treated with AKB-4924 when compared with unstimulated MSCs [(E), $n=3$ per group]. 
A

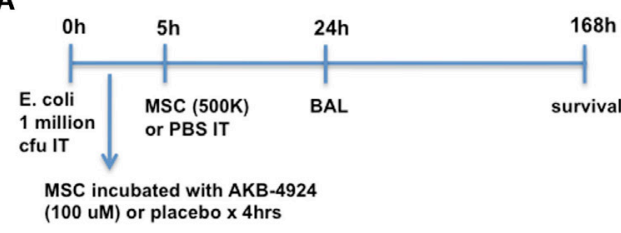

C

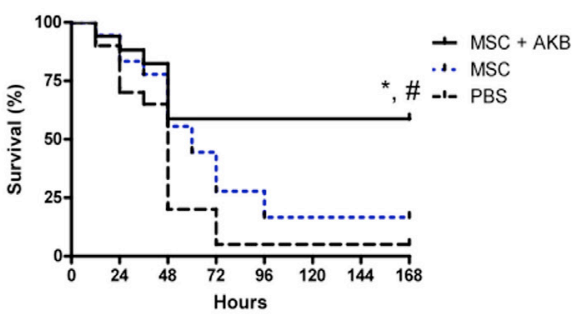

E

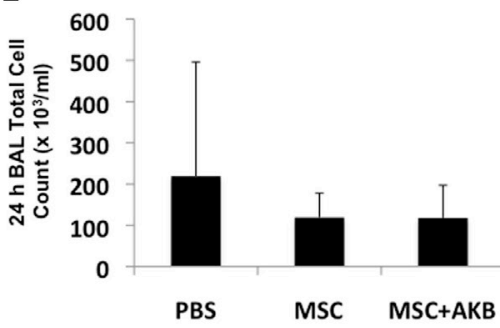

G

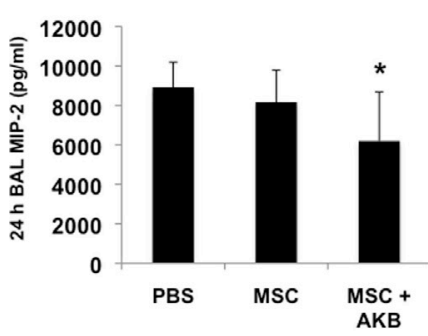

H

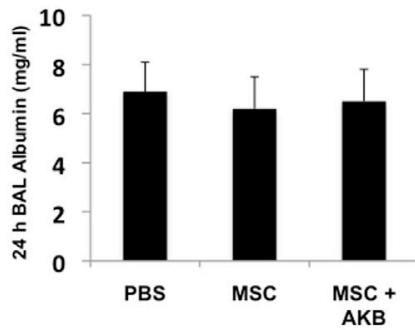

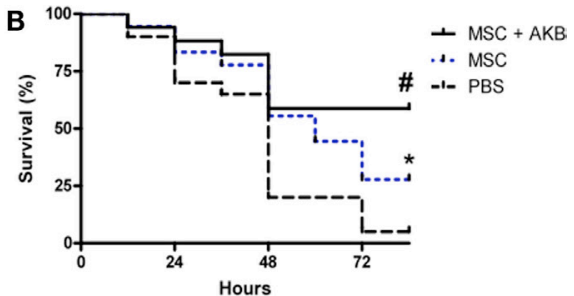

D

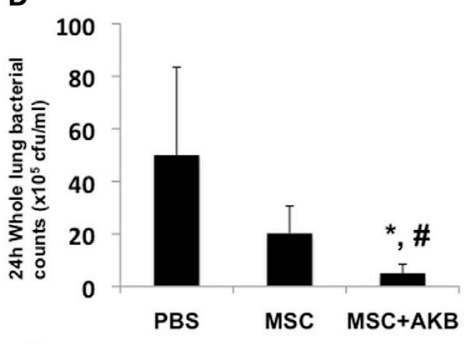

F

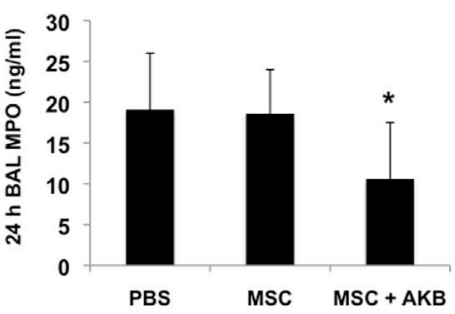

I

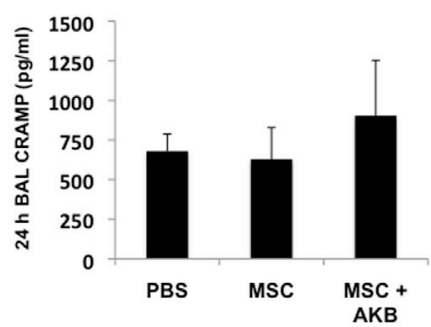

$\mathbf{K}$

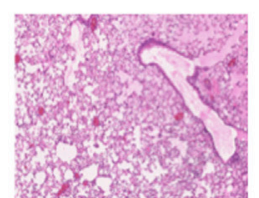

PBS

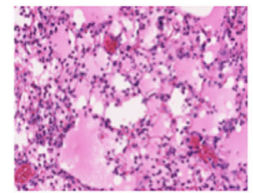

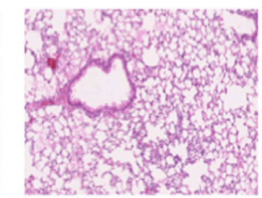

MSC

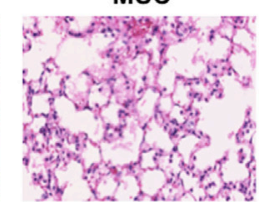

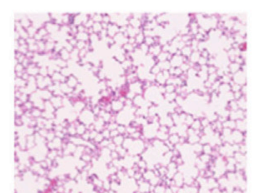

MSC + AKB

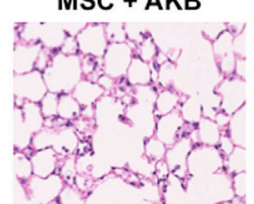

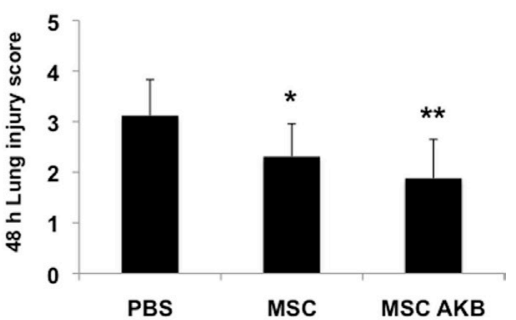

FIGURE 2 | AKB-4924 augments the therapeutic capacity of mesenchymal stem cells (MSCs) in an Escherichia coli pneumonia model. Following the experimental design outlined in panel (A), both unstimulated MSCs and MSCs pre-incubated with AKB-4924 (100 $\mu \mathrm{M} \times 4 \mathrm{~h})$ significantly improved the survival of mice at $72 \mathrm{~h}[(\mathbf{B}), \# p<0.05$ for MSC + AKB vs PBS, ${ }^{*} p<0.05$ for MSC vs PBS, $n=17-20$ per group], while only AKB-4924 stimulated MSCs increased survival over 7 days [(C), *, $p<0.05$ for MSC + AKB vs MSC and PBS treated groups, respectively, $n=17-20$ per group]. AKB-4924 also significantly improved the ability of MSCs to reduce whole lung bacterial burden [(D), **\# $p<0.05$ for MSC + AKB vs MSC and PBS treated groups, respectively, $n=5-6$ per group], alveolar neutrophil influx as measured by bronchoalveolar lavage (BAL) MPO levels [(F), ${ }^{*} p<0.05$ for MSC + AKB vs PBS treated group, $n=6-12$ per group], and inflammation as measured by BAL MIP-2 levels [(G), ${ }^{*} p<0.05$ for MSC + AKB vs PBS treated group, $n=6$ per group]. Total BAL cell counts [(E), $n=5$ per group], albumin concentration [(H), $n=5$ per group], and cathelicidin-related antimicrobial protein (CRAMP) levels [(I), $n=5$ per group] were not significantly changed in the BAL of mice treated with AKB-4924 stimulated MSCs. Lung injury was significantly reduced in both MSC and MSC + AKB treated groups, though the magnitude of improvement was greater in mice treated with AKB-4924 stimulated MSCs $\left[(\mathbf{J}, \mathbf{K}),{ }^{*} p<0.05\right.$ for MSC vs PBS treated group, ${ }^{* *} p<0.01$ for MSC + AKB vs PBS treated group, $n=8-12$ per group; images taken at 2.5 and $20 \times$ magnification]. 
(Figure 2H). BAL CRAMP was measured to see if it correlated with the reduction in bacterial burden seen in Figure 2D, but the increase in CRAMP observed with HIF-1 $\alpha$ stabilized MSCs did not reach statistical significance (Figure 2I). The improvements in bacterial clearance and inflammation were associated with a reduction in lung injury at $48 \mathrm{~h}$ postinfection, as assessed by histological methods, that was more pronounced in mice treated with HIF- $1 \alpha$ stabilized MSCs (Figures 2J,K).

\section{DISCUSSION}

Mesenchymal stem cells have been extensively studied as a potential therapy for severe lung injury and sepsis and have shown promise in several pre-clinical models (4-11). However, strategies to improve the survival of MSCs in inflammatory environments and thus augment their therapeutic potential are needed. This proof-of-principle study sought to enhance the therapeutic potential of MSCs in experimental lung injury due to pneumonia by stabilizing the transcription factor HIF$1 \alpha$ with the pharmacological agent AKB-4924. Results from this study substantiated our hypothesis by demonstrating that AKB-4924 improved: (a) MSC survival under in vitro cytotoxic conditions; (b) MSC antibacterial activity in vitro; and (c) MSC-derived therapeutic capacity in vivo with reduced mortality, bacterial burden, inflammation, and lung injury. Though, it is interesting to note that while BAL MPO levels were reduced, total BAL cell counts were not in this study. This discordance may due to a greater effect on neutrophil degranulation as opposed to absolute neutrophil recruitment to the alveolar space. Also, the lack of reduction in BAL albumin at $24 \mathrm{~h}$ is not concordant with the other parameters measured, which may be because it represents a summation of permeability over the entire time period and is not sensitive enough to detect changes that develop later in the timeframe being studied. Nevertheless, the overall findings suggest that methods to stabilize HIF- $1 \alpha$ in MSCs could be implemented in order to boost the therapeutic effect achieved in critically ill patients with lung injury, and are consistent with recent promising results in cardiac and vascular disease models (12-14).

Mesenchymal stem cells have been tested in several hundred clinical trials to date targeting a wide range of clinical diseases, but their clinical efficacy has not been reproducibly robust to date $(22,23)$. One of the potential explanations that has been suggested is the relatively short half-life of MSCs in vivo $(24,25)$. HIF- $1 \alpha$ represents an intuitive target to augment survival of MSCs in lung injury applications since the injured lung is a hypoxic environment requiring metabolic adaptations. Recent studies in experimental models of ischemia-reperfusion and radiation-induced lung injury have shown that hypoxic preconditioning of MSCs enhances their therapeutic efficacy $(26,27)$. The mechanisms demonstrated include improved MSC survival and antioxidant ability.

In this study, HIF- $1 \alpha$ stabilization in MSCs with the use of AKB-4924 resulted in significantly improved MSC survival under cytotoxic conditions and MSC-derived therapeutic capacity in vivo. While improving MSC survival is likely an important contributor to the augmented biological effect achieved with HIF-1 $\alpha$ stabilized MSCs, there are other possible mechanisms to consider. We provide some preliminary data that HIF-1 $\alpha$ stabilization augments the antibacterial property of MSCs, and it is possible that HIF- $1 \alpha$ stabilization in MSCs may be boosting other biological effects of MSCs such as growth factor secretion and immunomodulation. We also tested the possibility that HIF- $1 \alpha$ stabilization could keep MSCs in an undifferentiated, "stem-like" state that permits them to retain their reparative properties for a longer duration (28). However, screening qPCR analyses to determine if HIF- $1 \alpha$ stabilization upregulated-specific genes involved in maintaining an undifferentiated MSC phenotype (Oct4, TWIST) were unable to detect a significant difference compared with unstimulated MSCs (Figure S1 in Supplementary Material). Finally, HIF-1 $\alpha$ stabilized MSCs may be modulating the survival and function of other cell types that are known to be present in the injured lung such as alveolar epithelial cells, endothelial cells, neutrophils, and macrophages. These other potential mechanisms remain the focus of ongoing and future investigations.

While we used a small molecule, AKB-4924, to stabilize HIF- $1 \alpha$ in MSCs there are other potential methods that could be used to achieve this goal. Previous studies have used hypoxic preconditioning (i.e., growing MSCs under hypoxic conditions) to augment HIF- $1 \alpha$ expression. In addition, genetic editing could be applied to MSCs in order to inactivate the prolyl hydroxylase enzymes responsible for HIF- $1 \alpha$ degradation under normoxic conditions. However, genetic editing may carry an increased risk of malignant transformation of MSCs due to sustained dysregulation of HIF- $1 \alpha$ expression, particularly since HIF- $1 \alpha$ has been implicated in tumor development and invasiveness (29-31). In this regard, the use of AKB-4924 affords the advantage of stabilizing HIF- $1 \alpha$ for a defined time period that is determined by its own half-life. For acute inflammatory processes, such as lung injury due to bacterial pneumonia, even transient stabilization of HIF- $1 \alpha$ can lead to significant beneficial outcomes as we observed.

In summary, stabilization of HIF- $1 \alpha$ in MSCs, with the use of AKB-4924, significantly boosts MSC-derived therapeutic capacity in an E. coli model of bacterial pneumonia. Mechanistically, this may be due, in part, to improved MSC survival under cytotoxic conditions. This study and other recent publications suggest that strategies to stabilize HIF- $1 \alpha$ should be incorporated into MSC-based clinical trials for critically ill patients with lung injury.

\section{ETHICS STATEMENT}

All experiments were approved by the University of California, San Diego (UCSD) Institutional Animal Care and Use Committee (IACUC), and mice were housed in a UCSD facility approved by the Association for Assessment and Accreditation of Laboratory Animal Care (AAALAC). 


\section{AUTHOR CONTRIBUTIONS}

NG and VN planned the experiments, wrote the manuscript, and funded the studies. NG carried out the experiments.

\section{ACKNOWLEDGMENTS}

The authors thank Aerpio Therapeutics for supplying AKB-4924 to be utilized in this study. The study was supported by funding by

\section{REFERENCES}

1. Angus DC, Linde-Zwirble WT, Lidicker J, Clermont G, Carcillo J, Pinsky MR. Epidemiology of severe sepsis in the United States: analysis of incidence, outcome, and associated costs of care. Crit Care Med (2001) 29:1303-10. doi:10.1097/00003246-200107000-00002

2. World Health Organization. Antimicrobial Resistance: Global Report on Surveillance. Geneva (2014).

3. Bellani G, Laffey JG, Pham T, Fan E, Brochard L, Esteban A, et al. Epidemiology, patterns of care, and mortality for patients with acute respiratory distress syndrome in intensive care units in 50 countries. JAMA (2016) 315:788-800. doi:10.1001/jama.2016.0291

4. Xu J, Woods CR, Mora AL, Joodi R, Brigham KL, Iyer S, et al. Prevention of endotoxin-induced systemic response by bone marrow-derived mesenchymal stem cells in mice. Am J Physiol Lung Cell Mol Physiol (2007) 293:L131-41. doi:10.1152/ajplung.00431.2006

5. Gupta N, Su X, Popov B, Lee JW, Serikov V, Matthay MA. Intrapulmonary delivery of bone marrow-derived mesenchymal stem cells improves survival and attenuates endotoxin-induced acute lung injury in mice. J Immunol (2007) 179:1855-63. doi:10.4049/jimmunol.179.3.1855

6. Lee JW, Fang X, Gupta N, Serikov V, Matthay MA. Allogeneic human mesenchymal stem cells for treatment of E. coli endotoxin-induced acute lung injury in the ex vivo perfused human lung. Proc Natl Acad Sci U S A (2009) 106:16357-62. doi:10.1073/pnas.0907996106

7. Mei SH, Haitsma JJ, Dos Santos CC, Deng Y, Lai PF, Slutsky AS, et al. Mesenchymal stem cells reduce inflammation while enhancing bacterial clearance and improving survival in sepsis. Am J Respir Crit Care Med (2010) 182:1047-57. doi:10.1164/rccm.201001-0010OC

8. Gupta N, Sinha R, Krasnodembskaya A, Xu X, Nizet V, Matthay MA, et al. The TLR4-PAR1 axis regulates bone marrow mesenchymal stromal cell survival and therapeutic capacity in experimental bacterial pneumonia. Stem Cells (2018). doi:10.1002/stem.2796

9. McIntyre LA, Moher D, Fergusson DA, Sullivan KJ, Mei SH, Lalu M, et al. Efficacy of mesenchymal stromal cell therapy for acute lung injury in preclinical animal models: a systematic review. PLoS One (2016) 11:e0147170. doi:10.1371/journal.pone.0147170

10. Krasnodembskaya A, Song Y, Fang X, Gupta N, Serikov V, Lee JW, et al. Antibacterial effect of human mesenchymal stem cells is mediated in part from secretion of the antimicrobial peptide LL-37. Stem Cells (2010) 28:2229-38. doi:10.1002/stem.544

11. Nemeth K, Leelahavanichkul A, Yuen PS, Mayer B, Parmelee A, Doi K, et al. Bone marrow stromal cells attenuate sepsis via prostaglandin E2-dependent reprogramming of host macrophages to increase their interleukin-10 production. Nat Med (2009) 15:42-9. doi:10.1038/nm0409-462b

12. Cerrada I, Ruiz-Sauri A, Carrero R, Trigueros C, Dorronsoro A, SanchezPuelles JM, et al. Hypoxia-inducible factor 1 alpha contributes to cardiac healing in mesenchymal stem cells-mediated cardiac repair. Stem Cells Dev (2013) 22:501-11. doi:10.1089/scd.2012.0340

13. Liu XB, Wang JA, Ji XY, Yu SP, Wei L. Preconditioning of bone marrow mesenchymal stem cells by prolyl hydroxylase inhibition enhances cell survival and angiogenesis in vitro and after transplantation into the ischemic heart of rats. Stem Cell Res Ther (2014) 5:111. doi:10.1186/scrt499

14. HoWangYin KY, Loinard C, Bakker W, Guerin CL, Vilar J, d'Audigier C, et al. HIF-prolyl hydroxylase 2 inhibition enhances the efficiency of mesenchymal stem cell-based therapies for the treatment of critical limb ischemia. Stem Cells (2014) 32:231-43. doi:10.1002/stem.1540 the University of California, San Diego Department of Medicine (NG), and the National Institutes of Health/National Heart, Lung, and Blood Institute (HL125352, VN).

\section{SUPPLEMENTARY MATERIAL}

The Supplementary Material for this article can be found online at https://www.frontiersin.org/articles/10.3389/fmed.2018.00131/ full\#supplementary-material.

15. Okumura CY, Holands A, Tran DN, Olson J, Dahesh S, von Kockritz-Blickwede $\mathrm{M}$, et al. A new pharamacological agent (AKB-4924) stabiliizes hypoxia inducible factor-1 (HIF-1) and increases skin innate defenses against bacterial infection. J Mol Med (2012) 90:1079-89. doi:10.1007/s00109-012-0882-3

16. Leire E, Olson J, Isaacs $\mathrm{H}$, Nizet V, Hollands A. Role of hypoxia inducible factor-1 in keratinocyte inflammatory response and neutrophil recruitment. J Inflamm (2013) 10:28. doi:10.1186/1476-9255-10-28

17. Lin AE, Beasley FC, Olson J, Keller N, Shalwitz RA, Hannan TJ, et al. Role of hypoxia inducible factor- $1 \alpha$ in innate defense against uropathogenic Escherichia coli Infection. PLoS Pathog (2015) 11:e1004818. doi:10.1371/ journal.ppat.1004818

18. Polunovsky VA, Wendt CH, Ingbar DH, Peterson MS, Bitterman PB. Induction of endothelial cell apoptosis by TNF alpha: modulation by inhibitors of protein synthesis. Exp Cell Res (1994) 214:584-94. doi:10.1006/excr. 1994.1296

19. Tsuchida H, Takeda $Y$, Takei H, Shinzawa H, Takahashi T, Sendo F. In vivo regulation of rat neutrophil apoptosis occurring spontaneously or induced with TNF-alpha or cycloheximide. J Immunol (1995) 154:2403-12.

20. Mrozek JD, Smith KM, Bing DR, Meyers PA, Simonton SC, Connett JE, et al. Exogenous surfactant and partial liquid ventilation: physiologic and pathologic effects. Am J Respir Crit Care Med (1997) 156:1058-65. doi:10.1164/ ajrccm.156.4.9610104

21. Peyssonnaux C, Datta V, Cramer T, Doedens A, Theodorakis EA, Gallo RL, et al. HIF-1 alpha expression regulates the bactericidal capacity of phagocytes. J Clin Invest (2005) 115:1806-15. doi:10.1172/JCI23865

22. Squillaro T, Peluso G, Galderisi U. Clinical trials with mesenchymal stem cells: an update. Cell Transplant (2016) 25:829-48. doi:10.3727/ 096368915X689622

23. Trounson A, McDonald C. Stem cell therapies in clinical trials: progress and challenges. Cell Stem Cell (2015) 17:11-22. doi:10.1016/j.stem.2015. 06.007

24. Eggenhofer E, Luk F, Dahlke MH, Hoogduijn MJ. The life and fate of mesenchymal stem cells. Front Immunol (2014) 5:148. doi:10.3389/fimmu.2014. 00148

25. Li L, Chen X, Wang WE, Zeng C. How to improve the survival of transplanted mesenchymal stem cell in ischemic heart? Stem Cells Int (2016) 2016:9682757. doi:10.1155/2016/9682757.

26. Liu Y-Y, Chiang C-H, Hung S-C, Chian C-F, Tsai C-L, Chen W-C, et al. Hypoxia-preconditioned mesenchymal stem cells ameliorate ischemia/ reperfusion-induced lung injury. PLoS One (2017) 12:e0187637. doi:10.1371/ journal.pone. 0187637

27. Li B, Li C, Zhu M, Zhang Y, Du J, Xu Y, et al. Hypoxia-induced mesenchymal stromal cells exhibit an enhanced therapeutic effect on radiation-induced lung injury in mice due to an increased proliferation potential and enhanced antioxidant ability. Cell Physiol Biochem (2017) 44:1295-310. doi:10.1159/ 000485490

28. Tsai C-C, Chen Y-J, Yew T-L, Chen L-L, Wang J-Y, Chiu C-H, et al. Hypoxia inhibits senescence and maintains mesenchymal stem cell properties through down-regulation of E2A-p21 by HIF-TWIST. Blood (2011) 117:459-69. doi:10.1182/blood-2010-05-287508

29. Lu X, Kang Y. Hypoxia and hypoxia-inducible factors: master regulators of metastasis. Clin Cancer Res (2010) 16:5928-35. doi:10.1158/1078-0432. CCR-10-1360

30. Soni S, Padwad YS. HIF-1 in cancer therapy: two decade long story of a transcription factor. Acta Oncol (2017) 56:503-15. doi:10.1080/0284186X. 2017.1301680 
31. Qiu GZ, Jin MZ, Dai JX, Sun W, Feng JH, Jin WL. Reprogramming of the tumor in the hypoxic niche: the emerging concept and associated therapeutic strategies. Trends Pharmacol Sci (2017) 38:669-86. doi:10.1016/j. tips.2017.05.002

Conflict of Interest Statement: The authors declare that the research was conducted in the absence of any commercial or financial relationships that could be construed as a potential conflict of interest.
The reviewer AK declared a past co-authorship with one of the authors NG to the handling Editor.

Copyright $\odot 2018$ Gupta and Nizet. This is an open-access article distributed under the terms of the Creative Commons Attribution License (CC BY). The use, distribution or reproduction in other forums is permitted, provided the original author(s) and the copyright owner are credited and that the original publication in this journal is cited, in accordance with accepted academic practice. No use, distribution or reproduction is permitted which does not comply with these terms. 